\title{
Vascular compression of the left bronchus in a patient with funnel chest
}

\author{
Makoto Ando $^{1} \cdot$ Yuki Ichimori $^{1} \cdot$ Shigeru Sakamoto ${ }^{1}$
}

Received: 13 February 2020 / Accepted: 13 March 2020 / Published online: 24 March 2020

(c) The Japanese Association for Thoracic Surgery 2020

\begin{abstract}
We present a case of 5 year-old female with funnel chest, by which cardiovascular structures were displaced into the left chest cavity. This caused left bronchial compression by the pulmonary artery and the descending aorta, for which an external stenting was successfully performed. The patient had features suggestive of skeletal dysplasia with tracheobronchial malacia. One possible mechanism of vascular compression could be an association of fragility of the thoracic cavity and inspiratory dyspnea, causing thoracic deformity.
\end{abstract}

Keywords External stent $\cdot$ Vascular compression $\cdot$ Funnel chest $\cdot$ Pectus excavatum

\section{Introduction}

Airway obstruction resulting from a vascular compression syndrome, which is often associated with a cardiovascular abnormality, can cause repeat pneumonia and atelectasis [1]. Herein, we present a case with a suspected skeletal dysplasia, who exhibited left bronchial compression by the pulmonary artery and the descending aorta, for whom an external stenting (ES) [2] was performed. The potential mechanisms involved in the development of airway obstruction in this patient may be quite unique, and thus worth discussing.

\section{Case}

A female patient was born at the gestational age of 41 weeks. Her birth weight was $3.55 \mathrm{~kg}$. Her body height, head circumference, and chest circumference were $48.0 \mathrm{~cm}, 35.0 \mathrm{~cm}$, and $32.0 \mathrm{~cm}$, respectively. Her developmental milestone was uneventful until 1 year of age when she was diagnosed to have bronchial asthma. Although the body size at birth was within normal range, it gradually fell behind the mean value of the Japanese children. At 5 years, her body height was

Makoto Ando

shannon@kanazawa-med.ac.jp

1 Department of Cardiovascular Surgery, Kanazawa Medical University, 1-1 Daigaku, Uchinadamachi, Kahokugun, Ishikawaken 920-0293, Japan
$94.9 \mathrm{~cm}$ (standard deviation -2.7), and the body weight was $13.9 \mathrm{~kg}(-1.5)$. Her chest circumference was 46.0 (mean value 53.7$) \mathrm{cm}$. Her familial history was negative for delayed growth or puberty. Another notable feature was myopia and conspicuous funneling of the chest, which started developing around 2 years of age. She was noted to have a genu varum (bowlegs), with her knee joints $4.5 \mathrm{~cm}$ apart from each other while the ankles were together. Scoliosis or narrowing of the spines was not present, with the Cobb angle of $13^{\circ}$. The radiographic evaluation revealed that seven of eight carpal bones were ossified. There was no brachydactyly. There was no deformity of the skull, nor patent fontanelle and sutures. The skeletal maturity score, using the Tanner-Whitehouse method-2 [3] was 245; the number corresponds to the age of 63 months. Her blood chemistry data were within normal ranges. The hormone testing was notable for low insulin-like growth factor (somatomedin-C) level of 59 (normal 56-252 at 5 years) ng/ml. The short stature homeobox (SHOX) gene was negative.

For the respiratory symptom, she was found to have a left bronchial compression by the pulmonary artery and the descending aorta, for the first time at the age of 3 years on a chest computed tomography (CT) scan. Thereafter, she experienced a total of nine times of the hospitalization for pneumonia and was re-examined by the CT scan at the age of 4 years. The CT scan revealed an excavation of the chest resulting in a leftward shift of the cardiovascular structures, causing left bronchial compression (Fig. 1). Subsequently, she underwent a bronchoscopy under spontaneous breathing, 

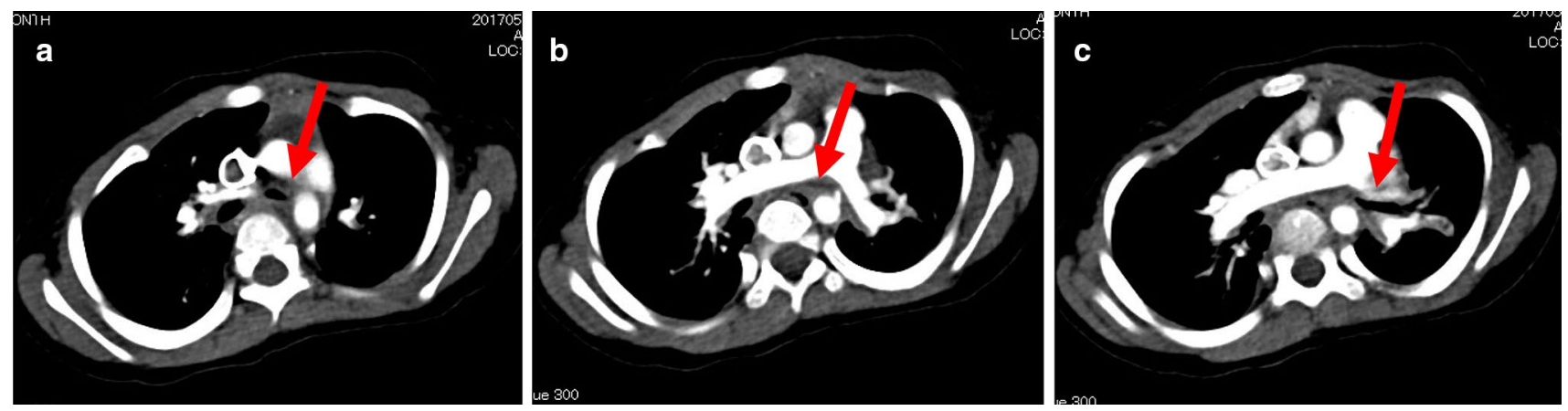

Fig. 1 Preoperative computer tomography scan images. There was an excavation of the chest resulting in a leftward shift of the cardiovascular structures, causing left bronchial compression. Images were proximal to (a), at the site of (b), and distal to(c) the compression. Red arrows indicate the sites of left bronchus

The aorta was first retracted away from the airway towards the chest wall using pledgeted sutures. A ringed PTFE prosthesis, $14 \mathrm{~mm}$ in size, was cut into two semicircular pieces. The midline stitches were placed and passed through the graft. The redundant ends of the graft were trimmed off and lateral ends of the cartilage were sutured to the graft. The other graft piece was fixed to the membrane by stitches placed along the midline. Both ends of the membrane stent were then trimmed off. The grafts were finally covered with a $0.1 \mathrm{~mm}$ PTFE sheet to prevent esophageal erosion (Fig. 2).

She was transferred to the intensive care unit and was weaned off the ventilator on day one. On a CT scan obtained on day 25 , the obstructed airway was widely patent (Fig. 3 ). The postoperative course was generally uneventful, and she was discharged from the hospital on day 31. After returning home, she had not experienced any episode of dyspnea nor pneumonia. The repeat CT scan at 3 months still revealed a widely patent airway. During the same admission, she underwent an arginine stimulation test to examine growth hormone secretion, which revealed the peak value of $13.4 \mu \mathrm{g} / \mathrm{L}$, excluding the presence of the hormone deficiency.
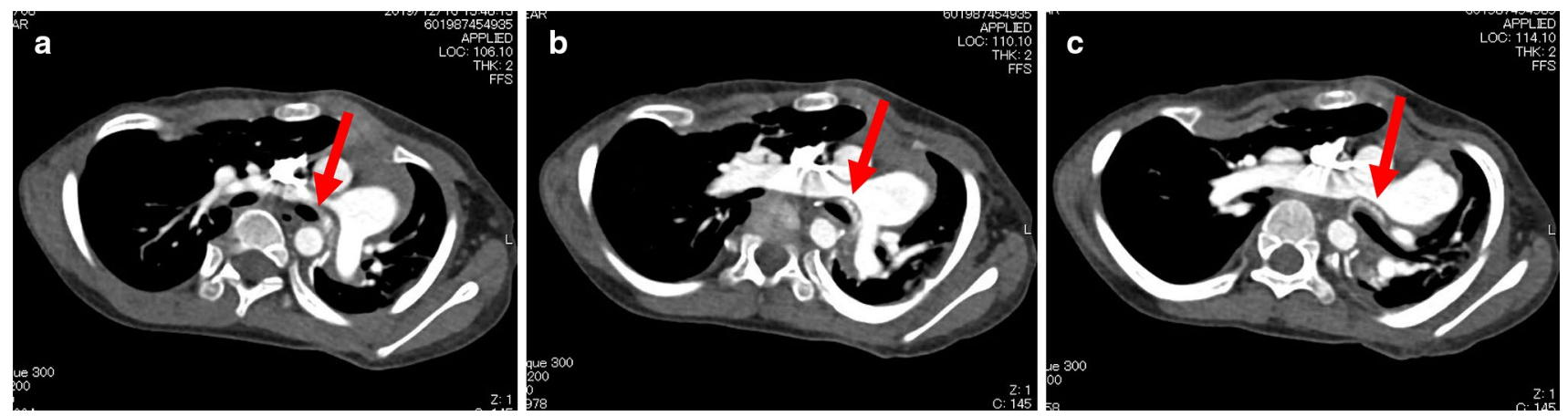

Fig. 3 Postoperative computer tomography scan image. The airway was widely patent proximal to (a), at the middle of (b), and at the distal end of (c) the stent. Red arrows indicate the sites of left bronchus and external stent 


\section{Discussion}

The first point of discussion regarding this case is the mechanism of vascular compression. Left bronchial compression by the descending aorta and the pulmonary artery, as was seen in this case, is the most frequently observed pattern of vascular compression. It typically develops after repair of the obstructive aortic arch. In this context, the descending aorta is shifted anteriorly and, thus, compress the airway against the anteriorly located pulmonary artery. On the contrary, the mechanism seen in the present case may be quite unique. On the CT scan, there was a severe funneling of the chest, causing the heart to be shifted leftward. This seemed to cause shifting of the pulmonary artery posteriorly, and finally compressed the left bronchus against the descending aorta. It draws interest that this patient had some features suggestive of skeletal dysplasia, such as short stature, genu varum, and myopia. It is known that a laryngotracheomalacia may associate skeletal dysplasia, such as craniocarpotarsal dysplasia, frontometaphyseal dysplasia, and Kniest dysplasia $[4,5]$. Considering these facts, her funnel chest, which gradually developed under the condition of tracheobronchial malacia, could be the effect of labored breathing-from airway malacia, bronchial stenosis, and pneumonia-on the fragile thoracic cavity.

The second point of discussion is the optimal treatment for this patient. Aortopexy is generally considered a main stem of the surgical repair [6] for vascular compression. However, in the situation of compression between the pulmonary artery and the descending aorta, aortopexy may be ineffective. Aortopexy is effective only if the other side of the airway to the vessel possess a fibrous continuity with a stable structure. One example of this is the tracheal compression by the anteriorly placed vessel. In this context, the trachea is firmly stabilized by the posterior fibrous tissue and, therefore, the anterior retraction of the vessel by the aortopexy expands the airway. This mechanism does not apply to the compression of the left bronchus, which is embedded in the soft and mobile lung. Moreover, some report its high failure rate due to an irreversible structural deformity of the cartilage [7], which was expected in the present case, in whom the bronchial compression had persisted for at least 2 years. ES, another option, is a unique surgical procedure to expand and stabilize the airway, by suspending its wall to the surrounding rigid prosthesis. It has been proven to be a reliable method to relieve airway compression in children [8], and was considered an optimal procedure for this patient. Presently, she enjoys her daily life almost free from respiratory symptom. However, follow-up of the skeletal dysplasia, whose presence remained to be identified, will be mandatory.
It is known that most of the cases with vascular compression are related to either congenital vascular anomaly and/or cardiovascular surgery. The present case differs from them, in that the funnel chest seemed to be the fundamental cause of compression. We thought that this report is valuable because of this unique mechanism of vascular compression that has never been reported. This study was approved by the institutional review board of Kanazawa Medical University, and ethical approval and written informed consent were waived. The authors have not received any support from any organization and, thus, have no conflicts of interest directly relevant to the content of this article.

\section{Compliance with ethical standards}

Conflict of interest There is no conflict of interest for the author or funding for the work.

\section{References}

1. Koivikko A, Puhakka HJ, Vikki P. Innominate artery compression syndrome. Presentation of 16 cases. ORL J Otorhinolaryngol Relat Spec. 1976;38:187-92.

2. Hagl S, Jakob H, Sebening C, van Bodegom P, Schmidt K, Zilow E, et al. External stabilization of long-segment tracheobronchomalacia guided by intraoperative bronchoscopy. Ann Thorac Surg. 1997;64:1412-20.

3. Tanner JM, Whitehouse RH, Cameron N, Marshall WA, Healy MJR, Goldstein H. Assessment of skeletal maturity and prediction of adult height (TW2 method). 2nd ed. London: Academic Press; 1983.

4. Moriwake T, Kawakami-Shinozuka M, Yasumitsu M, Nakamura T. Kniest dysplasia with severe tracheomalacia: bone dysplasia with respiratory complication. Clin Pediatr Endocrinol. 2005;14(Suppl 24):73-6.

5. Hicks J, De Jong A, Barrish J, Zhu SH, Popek E. Tracheomalacia in a neonate with kniest dysplasia: histopathologic and ultrastructural features. Ultrastruct Pathol. 2001;25:79-83.

6. Abdel-Rahman U, Ahrens P, Fieguth HG, Kitz R, Heller K, Moritz A. Surgical treatment of tracheomalacia in bronchoscopic monitors aortopexy in infant and children. Ann Thorac Surg. 2002;74:315-9.

7. Filler RM, Rossello PJ, Lebowitz RL. Life threatening anoxic spells caused by tracheal compression after repair of esophageal atresia: correction by surgery. J Pediatr Surg. 1976;11:739-48.

8. Ando M, Nagase Y, Hasegawa H, Takahashi Y. External stenting: a reliable technique to relieve airway obstruction in small children. J Thorac Cardiovasc Surg. 2017;153:1167-77.

Publisher's Note Springer Nature remains neutral with regard to jurisdictional claims in published maps and institutional affiliations. 